\title{
Higher Harmonics and Phase Contrast in Liquid Environment Dynamic Atomic Force microscopy - Tools for Observing Material Property Contrast in Biological Samples at High Resolution and Speed
}

Arvind Raman

School of Mechanical Engineering and the Birck Nanotechnology Center Purdue University, West Lafayette, IN 47907

Since its invention over 20 years ago dynamic Atomic Force Microscopy (dAFM) has become a major scientific technique for nanoscale surface imaging and force spectroscopy. One evolving important application of AFM is for the measurement and mapping of elasticity and adhesion (biomechanical assays) of biological samples including living cells in their native physiological liquid environments. Most commonly, researchers use static force-distance curves or the so-called "force-volume" mode for this purpose where the AFM tip indents and retracts point by point over the sample while recording the force as a function of separation. While this method has the advantage of providing quantitative information, it is quite slow, often applying larger imaging forces than in dynamic modes, and provides low resolution, highly pixilated images of local properties.

Recent advances in dynamic AFM in liquids have discovered new channels of highly sensitive local property information (higher harmonics, momentary excitation, bimodal excitation) [1-3] and forced a reinterpretation of some conventional channels (phase contrast) [4]. Observing these channels allows local material property mapping to be "piggy-backed" off the high speed and high resolution of tapping mode (or even frequency modulation) AFM. Using these channels of information it is possible to observe exquisite material property contrast of biological samples in buffer solutions. On the other hand, the conversion of this contrast to quantifiable local properties remains a challenge and an important topic of research. In this talk I will review the basic concepts behind these recent advances, illustrate the concepts with practical examples from the imaging of bacterial membranes, viruses, and living bacteria in buffer solutions, and discuss ongoing methods to make these data quantitative.

\section{References:}

[1] J. Preiner, J. L. Tang, V. Patsushenko, and P. Hinterdorfer, "Higher harmonic atomic force microscopy: Imaging of biological membranes in liquid", Physical Review Letters, 99, 046102, 2007. [2] N. F. Martinez, J. R. Lozano, E. T. Erruzo, F. Garcia, C. Richter, T. Sulzbach, R. Garcia, "Bimodal atomic force microscopy imaging of isolated antibodies in air and liquids", Nanotechnology, 19(38), $384011,2008$.

[3] X. Xu, J. Melcher, S. Basak, R. Reifenberger, A. Raman, “ Compositional contrast of biological materials in liquids using the momentary excitation of higher eigenmodes in dynamic atomic force microscopy“, Phys. Rev. Lett., 102(6), 060801, 2009.

[4] J. Melcher, C. Carrasco, X. Xu, J. L. Carrascosa, J. Gomez-Herrero, P. J. de Pablo, A. Raman, "Origins of phase contrast in the atomic force microscope in liquids", Proceedings of the National Academy of Sciences of the USA, (Direct submission), 106(33), 13655-13660, 2009. 


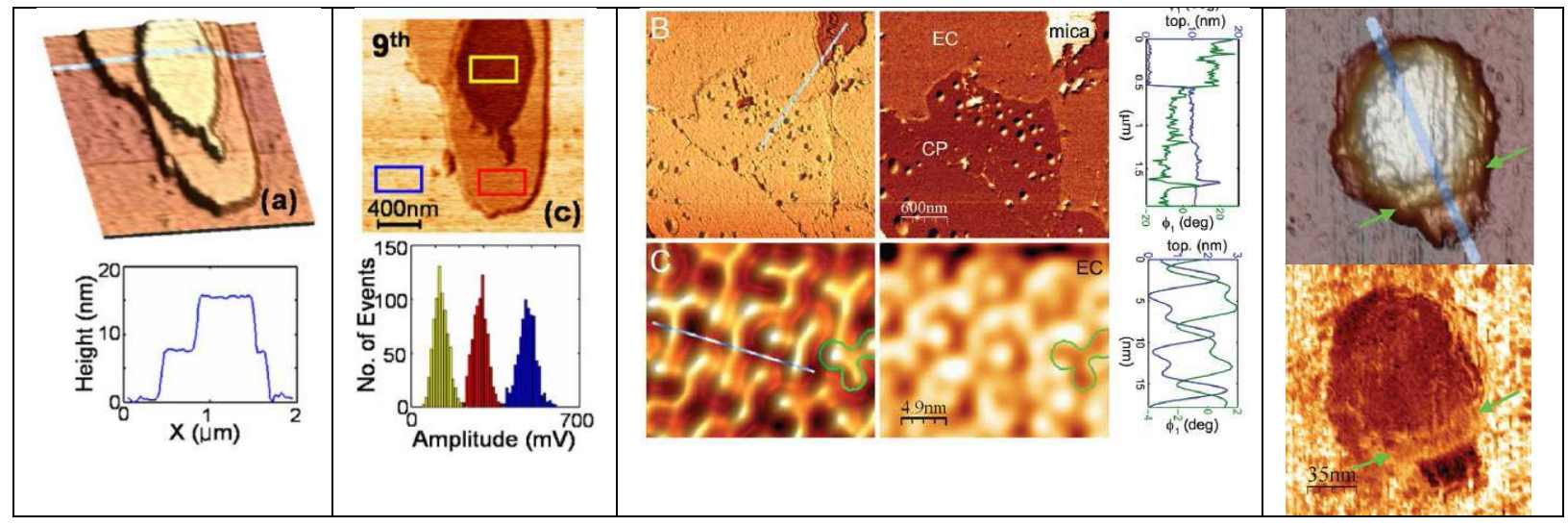

Examples illustrating new concepts in liquid environment tapping mode AFM:

on the left - momentary excited harmonics mapping showing how the natural ninth harmonic of cantilever drive can be used to distinguish between the stiffness of one vs. two layers of bacteriorhodopsin membrane. On the right are examples of topography and phase lag maps of purple membrane showing that energy dissipation (phase lag) is greater on stiffer/non-dissipative substrates counter to what would be expected in air. 